\title{
Mobile, awake and critically ill
}

\section{Margaret S. Herridge MD MPH}

$\infty$

\author{
See related article page $69 \mathrm{I}$
}

I $\mathrm{n}$ this issue of $C M A J$, Clavet and colleagues present a comprehensive evaluation of the prevalence and risk factors for joint contractures in a large cohort of patients with critical illness. ${ }^{1}$ The title of my commentary may seem improbable, yet the article by Clavet and investigators highlights an important and previously under-recognized consequence of immobility caused by prolonged critical illness. Joint contractures and their contribution to long-term functional disability represent a small part of the problem as we continue to document the varied and often staggering morbidities associated prolonged severe critical illness.

Clavet and colleagues showed that more than one-third of patients with a prolonged stay of 2 or more weeks in an intensive care unit (ICU) each had at least 2 functionally significant joint contractures. Furthermore, at the time of hospital discharge, $\mathrm{I}$ in 4 patients had contractures that were still present and disrupted their daily activities. The major risk factor for the development of contractures was length of ICU stay, and the odds of acquiring a contracture increased markedly between weeks 2 and 8 in the ICU. Despite the limitations of this retrospective cohort design, including chart-derived data, a single study centre and the potential for missing or absent data for other possible confounding variables, it is difficult not to be impressed by the rigor of this investigation and the magnitude of its findings.

There is robust literature from many investigators in different countries and involving diverse patient populations that demonstrates important impairments in health-related quality of life among patients who have had a critical illness. These decrements in quality of life may persist for years and may be irreversible in some cases. ${ }^{2-8}$ Contractures acquired during a prolonged ICU stay are another important contributor to decreased quality of life. Upper-extremity contractures can dramatically limit many essential activities of daily living, such as bathing, toileting and grooming, and lowerextremity contractures can limit walking or promote falling, and they may impede a patient's ability to return to work if his or her occupation is physically demanding. In addition, all physical leisure activities will be compromised to some extent. As a direct consequence of this impaired mobility, caregivers must assume a greater burden of responsibility, and this has important implications for their mental and physical health status. ${ }^{9-11}$

Large joint contractures can now be added to the growing list of physical and neuropsychological sequelae that follow critical illnesses (Box I). There is considerable heterogeneity in outcomes across different patient populations. It is difficult but imperative that we continue to study the relations be-

\section{Key points of the article}

- The success of intensive care medicine has lead to large numbers of patients discharged from the ICU with important physical and neuropsychological disabilities.

- The most common morbidity after critical illness is a muscle wasting and weakness syndrome related to nerve and muscle injury acquired during critical illness.

- A cultural shift in the ICU to greater mobility and increased wakefulness may decrease the morbidity of the ICU stay.

tween these outcomes and different patient demographics, underlying disease states and processes of care delivery to determine contributing risk factors that may be eliminated or modified.

There appears to be significant potential for harm arising from the current ICU culture of patient immobility and an often excessive or unnecessary use of sedation. This is a culture that began for all the right reasons: to promote patient comfort, safety and respiratory synchrony while allowing intubation and mechanical ventilatory support for severe respiratory failure. But this culture has persisted despite emerging evidence that these current practices may, alone or in combination with the acute illness that precipitated the ICU admission, have important adverse consequences that may not be remediable over time. Immobility clearly plays a role in the development of contractures, and immobility is also a contributing factor to the ubiquitous finding of profound muscle wasting and weakness among patients who have long ICU stays and may contribute to their inability to regain premorbid functional status.

The current literature supports that patients in critical care settings may be mobilized without harm, even while receiving mechanical ventilation, and that wakefulness is safe and may be associated with improved neuropsychological outcomes. ${ }^{15-17}$ A recent study demonstrated how spontaneous awakening, when combined with a spontaneous breathing trial, was associated not only with a decreased duration of mechanical ventilation and ICU length of stay but also with improved survival in the first year after ICU discharge. ${ }^{18}$ The patients in the study by Clavet and colleagues had exposure to I.25 full-time physiotherapists, one 0.75 full-time occupational therapist and a full-time nursing staff who routinely repositioned the patients. It is not clear what

Margaret Herridge is with the Department of Respiratory and Critical Care Medicine, University Health Network, University of Toronto, Toronto, Ont. 
Box 1: Physical and neuropsychological sequelae of critical illness ${ }^{6,7,12-14}$

\section{Physical}

- Muscle wasting and weakness (critical illness neuromyopathy)

- Entrapment neuropathy (foot and wrist drop)

- Peripheral nerve injury (persistent numbness, paresthesia)

- Heterotopic ossification

- Hoarseness, voice change

- Tracheal stenosis

- Dysphagia

- Changes in taste

- Cosmetic changes (transient alopecia, scars from tracheostomy, chest tubes, central line sites, thoracotomy, laparotomy, striae from volume overload)

- Sexual dysfunction

- Contractures

Neuropsychological

- Delirium

- Cognitive dysfunction (impairments in memory, attention, concentration, executive functioning)

- Anxiety, depression

- Post-traumatic stress disorder

- Sleep disruption or disturbances

- Panic attack disorder

the optimal staffing ratios should be in an ICU setting, but clearly for patients to fully benefit from the services of allied health professionals in the ICU, they need to be awake, cooperative and mobile.

Although the authors failed to identify a subgroup at increased risk for contractures or muscle wasting and weakness, such subgroups warrant further scrutiny. As there are increasingly older patients occupying critical care beds, we need to understand how multiple comorbidites and preexisting brain or neuromuscular dysfunction modify the ability of patients to withstand the additional insult of prolonged immobility and exposure to psychoactive medications (the socalled "disease of critical illness"). Equally, we have a responsibility to understand patients' innate resilience following critical illness and their ability to return to an acceptable quality of life and functional independence. ${ }^{19,20}$ Detailed knowledge of these outcomes may inform decisions to prioritize certain vulnerable patient subsets for early mobility and rehabilitation during their ICU stay, which may have practical importance in the context of limited allied health resources.

The physical and neuropsychological consequences of an ICU stay represent a burgeoning public health issue that has not been adequately addressed. As our population ages, there will be many patients discharged from the ICU with disability. Currently, there is no provision for the long-term needs of these patients, no systematic follow-up, no clear understanding of the pathophysiologic mechanisms underlying many of the most prevalent morbidities and no known intervention that reliably improves outcomes apart from a self-administered educational manual. ${ }^{21}$ As a society, we have chosen to invest heavily in the acute care needs of critically ill patients. Let us now equally invest in optimizing outcomes for the survivors and caregivers of this costly and arduous intervention.

Competing interests: None declared.

\section{REFERENCES}

I. Clavet H, Hébert PC, Fergusson D, et al. Joint contracture following prolonged stays in the intensive care unit. CMAJ 2008:178:69I-7.

2. McHugh LG, Milberg JA, Whitcomb ME, et al. Recovery of function in survivors of the acute respiratory distress syndrome. Am J Respir Crit Care Med I994;150:90-4.

3. Weinert CR, Gross CR, Kangas JR, et al. Health-related quality of life after acute lung injury. Am J Respir Crit Care Med I997;156:1120-8.

4. Davidson TA, Caldwell ES, Curtis JR, et al. Reduced quality of life in survivors of acute respiratory distress syndrome compared with critically ill control patients. JAMA 1999;281:354-60.

5. Angus DC, Musthafa AA, Clermont G, et al. Quality-adjusted survival in the first year after the acute respiratory distress syndrome. Am J Respir Crit Care Med 200I; I63:1389-94.

6. Herridge MS, Cheung AM, Tansey CM, et al. One-year outcomes in survivors of the acute respiratory distress syndrome. N EngIJ Med 2003;348:683-93.

7. Hopkins RO, Weaver LK, Collingridge D, et al. Two-year cognitive, emotional, and quality of life outcomes in acute respiratory distress syndrome. Am J Respir Crit Care Med 2005; I71:340-7.

8. Cheung AM, Tansey CM, Tomlinson G, et al. Two year outcomes, costs and health care utilization in survivors of ARDS. Am J Respir Crit Care Med 2006;174:538-44.

9. Van Pelt DC, Milbrandt EB, Qin L, et al. Informal caregiver burden among survivors of prolonged mechanical ventilation. Am J Respir Crit Care Med 2007;175:167-73.

Io. Cameron JI, Herridge MS, Tansey CM, et al. Caregiver burden in survivors of the acute respiratory distress syndrome. Crit Care Med 2006;34:8I-6.

II. Azoulay E, Pochard F, Kenish-Barnes N, et al. Risk of Post traumatic stress symptoms in family members of intensive care unit patients. Am J Respir Crit Care Med 2005;I7I:987-94.

I2. Griffiths $\mathrm{RD}$, Jones C. Recovery from intensive care. BMJ I999;319:427-9.

I3. Herridge MS, Batt J, Hopkins RO. The pathophysiology of long-term outcomes following critical illness. Crit Care Clin 2008;24:179-99.

I4. Ely EW, Shintani A, Truman B, et al. Delirium as a predictor of mortality in mechanically ventilated patients in the intensive care unit. JAMA 2004;29I:1753-62.

I5. Kress JP, Gehlbach B, Lacy M, et al. The long-term psychological effects of daily sedative interruption on critically ill patients. Am J Respir Crit Care Med 2003; 68 I457-6I.

I6. Jones C, Griffiths RD, Humphries G, et al. Memory, delusions, and the development of acute posttraumatic stress disorder-related symptoms after intensive care. Crit Care Med 200I;29:573-80.

I7. Morris PE. Moving our critically ill patients: mobility barriers and benefits. Crit Care Clin 2007;23:I-20.

I8. Girard TD, Kress JP, Fuchs BD, et al. Efficacy and safety of a paired sedation and ventilator weaning protocol for mechanically ventilated patients in intensive care (Awakening and Breathing Controlled trial): a randomized controlled trial. Lancet 2008;371:126-34.

I9. Dutta C, Hadley EC. The significance of sarcopenia in old age. J Gerontol A Biol Sci Med Sci I995;50:I-4.

20. Janssen I, Shepard DS, Katzmarzyk PT, et al. The health care costs of sarcopenia in the United States. J Am Geriatr Soc 2004;52:80-5.

2I. Jones C, Skirrow P, Griffiths RD, et al. Rehabilitation after critical illness: a randomized, controlled trial. Crit Care Med 2003;31:2456-6I.

Correspondence to: Dr. Margaret S. Herridge, Respiratory and Critical Care Medicine, University Health Network, University of Toronto, IIC-II80 New Clinical Services Building, 585 University Ave. Toronto ON M5G 2N2; fax 416 340-3Io9;

margaret.herridge@uhn.on.ca 